\title{
Height-unmixed of Tensor Product of Lattices
}

\author{
Ali Molkhasi \\ Department of Mathematics, Faculty of Mathematical Sciences, University of Farhangian, Tabriz, Iran
}

Copyright (C) 2016 by authors, all rights reserved. Authors agree that this article remains permanently open access under the terms of the Creative Commons Attribution License 4.0 International License

\begin{abstract}
We investigate WB-height-unmixed of tensor product of distributive lattices: Cohen-Macaulay rings related to tensor products of distributive lattices are constructed using the method of Stanly and Reisner.
\end{abstract}

Keywords Distributive Lattices, Tensor Product, CohenMacaulay Rings, Stanley-Reisner Ring, Unmixedness

2010 AMS(MOS) Subject Classification: $13 \mathrm{H} 10$, 08B10, 08B25

\section{Introduction}

All rings and algebras considered in this paper are commutative with identity elements and $k$ stands for a perfect field. Suitable background on depth of algebraic geometry and Cohen-Macaulay rings is [3], [8], and [11].

We recall that tensor products were introduced in $\mathrm{J}$. Anderson and N. Kimura [1] and G. A. Fraser [5]. Our aim in this paper is to prove that the Cohen-Macaulay property is inherited by tensor products of $k$-algebras and tensor products of distributive lattices. Tensor products of semilattices and related structures have already been the source of many publications ([5] and [7]). We denote by $A \otimes B$ the tensor product of $A$ and $B$, which $A$ and $B$ are $\{\vee, 0\}$-semilattices.

In this paper, we suppose $k$ is a perfect field and $F$ and $K$ be two extension fields of $k$ such that $F \otimes K$ is Noetherian. Then we prove that if $L_{1}$ and $L_{2}$ are distributive lattices, then the ring $(F \otimes K)\left[L_{1} \otimes L_{2}\right]\left[X_{1}, X_{2}, \ldots\right]$ is WB-heightunmixed. Finally, WB-height-unmixed is related to universally catenary and approximately Cohen-Macaulay ring.

\section{Tensor Product and Cohen- Macaulay Rings}

Let $A$ be a lattice. If $a, b \in A$, the join and meet of $a$ and $b$ are written as $a \vee b$ and $a \wedge b$, respectively. If $a_{i} \in A$ for $i \in I$, where $I$ is any non-empty set, then the join and meet of $\left\{a_{i}: i \in I\right\}$, if they exist, are denoted by $\vee_{i \in I} a_{i}$ and $\wedge_{i \in I} a_{i}$, respectively. The smallest and largest elements of $A$, if they exist, are denoted by 0 and 1 , respectively. Tensor products of vector spaces are well-known, but it exists in many other categories equipped with a forgetful functor to Set [14]. Examples of such categories that matters to us are boolean algebra, distributive lattices, semilattices with zero, etc. Given $A, B$ and $X$, three object of the same category, a bimorphism from $A \times B$ to $X$ is a set theoretic map $f: A \times B \longrightarrow X$ such that for all $a \in A$ and for all $b \in B$, the mappings $f(a,-): B \longrightarrow X$ and $f(-, b): A \longrightarrow X$ are morphisms. Being given an object $X$ of the category and a bimorphism $i: A \times B \longrightarrow X$, we say that $X$ is a tensor product of $A \times B$ if for every object $C$ and every bimorphism $f: A \times B \longrightarrow C$, there exist a unique morphism $h: X \longrightarrow C$, such that $f=h \circ i$. Tensor products are unique up to isomorphisms and they are denoted by $A \otimes B$. The bimorphism $i$ is not surjective but its image generates $A \otimes B$, thus we call generating elements (of $A \otimes B$ ) those coming from $A \otimes B$ and we will write $i(a, b)=a \otimes b$. Now, we have the following definitions from [5].

Definition 2.1. Let $A, B$ and $C$ be distributive lattices. A function

$$
f: A \times B \longrightarrow C
$$

is a bihomomorphism if the functions $g_{a}: B \longrightarrow C$ defined by $g_{a}(b)=f(a, b)$ and $h_{b}: A \longrightarrow C$ defined by $h_{b}(a)=$ $f(a, b)$ are homomorphisms for all $a \in A$ and $b \in B$.

Definition 2.2. Let $A$ and $B$ be distributive lattices. A distributive lattice $C$ is a tensor product of $A$ and $B$ (in the category $\mathfrak{D})$, if there exists a bihomomorphism $f: A \times B \longrightarrow C$, such that $C$ is generated by $f(A \times B)$ and for any distributive lattice $D$ and any bihomomorphism $g: A \times B \longrightarrow D$, there is a homomorphism $h: C \longrightarrow D$ satisfying $g=h f$. 
Note that since $f(A \times B)$ generates $C$, the homomorphism $h$ is necessarily unique. Let $A$ and $B$ be distributive lattices. Then a tensor product of $A$ and $B$ in the category $\mathfrak{D}$ exists and is unique up to isomorphism (see [5]).

The tensor product of $A$ and $B$ is denoted by $A \otimes B$ and the image of $(a, b)$ under the canonical bihomomorphism $f$ : $A \times B \longrightarrow A \otimes B$ is written as $a \otimes b$. Now, $A \otimes B$ is the distributive lattice generated by the elements $a \otimes b(a \in$ $A, b \in B$ ), subject to the bihomomorphic conditions:

$$
\begin{aligned}
& \left(a_{1} \vee a_{2}\right) \otimes b=\left(a_{1} \otimes b\right) \vee\left(a_{2} \otimes b\right), \\
& \left(a_{1} \wedge a_{2}\right) \otimes b=\left(a_{1} \otimes b\right) \wedge\left(a_{2} \otimes b\right), \\
& a \otimes\left(b_{1} \vee b_{2}\right)=\left(a \otimes b_{1}\right) \wedge\left(a \otimes b_{2}\right),
\end{aligned}
$$

and

$$
a \otimes\left(b_{1} \wedge b_{2}\right)=\left(a \otimes b_{1}\right) \wedge\left(a \otimes b_{2}\right),
$$

for all $a, a_{1}, a_{2} \in A$ and $b, b_{1}, b_{2} \in B$. Every element of $A \otimes B$ can be written in the form $\vee_{i=1}^{n}\left(a_{i} \otimes b_{i}\right)$ for some $a_{i} \in I$ and $b_{i} \in B, i=1,2, \ldots, n$.

For terminology and basic results of lattice theory and universal algebra, consult Birkhoff [4] and Gratzer [10]. In this paper, relationship among tensor product of the Cohen-Macaulay rings, tensor product of the distributive lattices, WB-height-unmixed and Stanley-Reisner ring are considered.

We first recall the definition of an algebra with straightening lows in [6], then recall WB-height-unmixed in ideal of a commutative ring in [11].

Definition 2.3. Given a commutative ring $R$ with identity element. If $P$ is a finite partially ordered set, then we say that $\mathfrak{A}$ is a $A S L$ (algebra with straightening lows) on $P$ over $R$ if the followings hold:

$A S L$ - 0 . An injective map $P \hookrightarrow \mathfrak{A}$ is given, $\mathfrak{A}$ is a graded $R$-algebra generated by $P$, and each element of $P$ is a homogeneous of positive degree. We call a product of elements of $P$ a monomial in $P$. In general, a monomial $M$ is a map $P \rightarrow \mathbb{N}_{0}$ and we denote $M=\prod_{x \in p} x^{M(x)}$ such that it also stands for an element of $\mathfrak{A}$. A monomial in $P$ of the form

$$
x_{i_{1}} \cdots x_{i_{l}}
$$

with $x_{i_{1}} \leq \cdots \leq x_{i_{l}}$ is called standard.

$A S L-1$. The set of standard monomials in $P$ is an $R$-free basis of $\mathfrak{A}$.

$A S L-2$. For $x, y \in P$ that $x \not \leq y$ and $y \not \leq x$, there is an expression of the form

$$
x y=\sum_{M} c_{M}^{x y} M\left(c_{M}^{x y} \in R\right)
$$

where the sum is taken over all standard monomials

$$
M=x_{1} \cdots x_{r_{M}} \quad\left(x_{1} \leq \cdots \leq x_{r_{M}}\right)
$$

with $x_{1}<x, y$ and $\operatorname{deg} M=\operatorname{deg}(x y)$.

The most simple example of an $A S L$ on $P$ over $R$ is the Stanley-Reisner ring $R[P]=R[x \mid x \in P] /(x y \mid x \not \leq y$, $y \nless \leq x)$. The Stanley-Reisner rings play central role in theory of $A S L$. For the proof of the following theorem, see [6].

Theorem 2.4. If $R$ is Cohen-Macaulay ring, and if $P$ is a distributive lattice, then $R[P]$ is Cohen-Macaulay.

Let $I$ is ideal of the ring $R$. Then we say that an ideal $I$ is unmixed if $I$ has no embedded prime divisors or, in modern language, if the associated prime ideals of $R / I$ are the minimal prime ideals of $I$. We know a prime ideal $\mathfrak{p}$ is a weak Bourbaki associated prime of the ideal $I$ of the ring $R$ if for some $a \in R$ it is a minimal ideal of the form $I: a$.

We recall that an ideal is WB-height-unmixed, if it is height-unmixed with respect to the set of weak Bourbaki associated primes, which an ideal is height-unmixed if all the associated primes of $I$ have equal height.

Before proving main theorem let us note that a ring is regular if for all prime ideal $\mathfrak{p}$ of $R, R_{\mathfrak{p}}$ is a regular local ring. Recently, in [2], Bouchiba and Kabbaj showed that if $R$ and $S$ are $k$-algebras such that $R \otimes_{k} S$ is Noetherian then $R \otimes_{k} S$ is a Cohen-Macaulay ring if and only if $R$ and $S$ are Cohen-Macaulay rings. Now we are ready to present our main theorem.

Theorem 2.5. Let $k$ be a perfect field, $F$ and $K$ be two extension fields of $k$ such that $F \otimes K$ is Noetherian. If $L_{1}$ and $L_{2}$ are distributive lattices, then the ring $(F \otimes K)\left[L_{1} \otimes\right.$ $\left.L_{2}\right]\left[X_{1}, X_{2}, \ldots\right]$ is WB-height-unmixed.

Proof. By ([4], Theorem 2.6), $L_{1} \otimes L_{2}$ is distributive lattice if $L_{1}$ and $L_{2}$ are distributive lattices. On the other hand, in [15] and [2], it is proved that the tensor product of two extension fields of $k$ is not necessarily Noetherian. Here, for all two $k$-algebras $F$ and $K$ we have $F \otimes K$ is Noetherian and $k$ is perfect field. From the note on page 49 of [13] we will have that $F \otimes K$ is regular ring. By using the following well-known chain we conclude $F \otimes K$ is Cohen-Macaulay ring:

$$
\begin{aligned}
\text { Regular } & \Longrightarrow \text { Complete intersection } \Longrightarrow \text { Gorenstein } \\
& \Longrightarrow \text { Cohen Macaulay }
\end{aligned}
$$

On the other hand, by applying that Theorem 2.4 , if $R$ is Cohen-Macaulay and $P$ is distributive lattice, then $R[P]$ is Cohen-Macaulay ring. Here, we will have $(F \otimes K)\left[L_{1} \otimes\right.$ $\left.L_{2}\right]$ is Cohen-Macaulay ring. By ([12], Theorem 2.3), $(F \otimes$ $K)\left[L_{1} \otimes L_{2}\right]\left[X_{1}, X_{2}, \ldots\right]$ is WB-height-unmixed. 
We will say that a Noetherian ring $R$ is catenary if every saturated chain joining prime ideals $\mathfrak{p}$ and $\mathfrak{q}$ has (maximal) length height $\mathfrak{q} / \mathfrak{p}$ such that $\mathfrak{p} \subseteq \mathfrak{q}$. Also, we say that $R$ is universally catenary if all the polynomial rings $R\left[X_{1}, X_{2}, \ldots\right]$ are catenary.

Theorem 2.6. Let $k$ be a perfect field, $F$ and $K$ be two extension fields of $k$ such that $F \otimes K$ is Noetherian. If $L_{1}$ and $L_{2}$ are distributive lattices, then the ring $(F \otimes K)\left[L_{1} \otimes L_{2}\right]$ is universally catenary.

Proof. In Theorem 2.5, we showed that $(F \otimes K)\left[L_{1} \otimes L_{2}\right]$ Cohen-Macaulay ring. By applying that ([3], Theorem 2.1.12), we see that every Cohen-Macaulay ring is universally catenary. Therefore, $(F \otimes K)\left[L_{1} \otimes L_{2}\right]$ is universally catenary.

Corollary 2.7. Let $k$ be a perfect field, $F$ and $K$ be two extension fields of $k$ such that $F \otimes K$ is Noetherian. If $L_{1}$ and $L_{2}$ are distributive lattices, then any polynomial algebra over $(F \otimes K)\left[L_{1} \otimes L_{2}\right]$ is Cohen-Macaulay ring.

Corollary 2.8. Let $k$ be a perfect field, $F$ and $K$ be two extension fields of $k$ such that $F \otimes K$ is Noetherian. If $L_{1}$ and $L_{2}$ are distributive lattices, then any polynomial algebra over $(F \otimes K)\left[L_{1} \otimes L_{2}\right]$ is universally catenary.

Corollary 2.9. Let $k$ be a perfect field, $F$ and $K$ be two extension fields of $k$ such that $F \otimes K$ is Noetherian. If $L_{1}$ and $L_{2}$ are distributive lattices, then any quotient of $(F \otimes$ $K)\left[L_{1} \otimes L_{2}\right]$ is universally catenary.

Now, assume that $(R, \mathfrak{m})$ is local ring with $\operatorname{dim}(R)=d$. We say that $R$ is an approximately Cohen-Macaulay ring if either $\operatorname{dim}(R)=0$ or there exists an element $r$ of $\mathfrak{m}$ such that $R / a^{n} R$ is a Cohen-Macaulay ring of dimension $d-1$ for every integer $n>0$ ([9]). A ring $R$ is called an approximately Cohen-Macaulay ring if the ring $R_{\mathfrak{p}}$ is an approximately Cohen-Macaulay ring, for all prime ideals $\mathfrak{p}$ of $R$.

Corollary 2.10. Let $k$ be a perfect field and $F$ and $K$ be nonzero $k$-algebras such that $F \otimes K$ is Noetherian. Assume that $F$ is not a Cohen-Macaulay ring. If $F \otimes K$ is an approximately Cohen-Macaulay ring, then $K\left[X_{1}, X_{2}, \ldots\right]$ is universally catenary.

\section{Acknowledgements}

The author is deeply grateful of Professor I. Zamani for his careful reading of the paper and valuable suggestions.

\section{REFERENCES}

[1] J. A. Anderson and N. Kimura, The tensor product of semilattices, Semigroup Forum, 16 (1978), 83-88.

[2] S. Bouchiba and S. Kabbaj, Tensor products of CohenMacaulay rings Solution to a problem of Grothendieck, Journal of Algebra, 252 (2002), 65-73.

[3] W. Bruns and J. Herzog, Cohen-Macaulay rings, Cambridge University Press, Cambridge, 1997.

[4] G. Birkhoff, Lattice theory, 3rd ed. (Colloq. Publ., Vol. 25, Amer. Math. Soc, Providence, R.I., 1967).

[5] G. A. Fraser, The semilattice tensor product of distributive semilattices, Trans. Amer. Math. Soc., 217 (1976), 183-194.

[6] C. De Concini and D. Eisenbud, Hodge algebras, asterisque, societe mathematique de france, 91 (1982).

[7] G. Gratzer and F. Wehrung, Tensor products of semilattices with zero, Journal of Pure and Applied Algebra, 147 (2000), 273-301.

[8] R. Gilmer, Multiplicative ideal theory, Marcel Dekker, New York, 1972.

[9] S. Goto, Approximately Cohen-Macaulay Rings, J. Algebra 76(1) (1982), 214-225.

[10] G. Gratzer, Universal algebra, Van Nostrand, Princeton, N.J., 1968.

[11] I. Kaplansky, Commutative ring theory, University of Chicago Press, Chicago, 1974.

[12] A. Molkhasi, Polynomials, $\alpha$-ideals and the principal lattice, Journal of Siberian Federal University, 4(3) (2011), 292-297.

[13] R. Y. Sharp, Simplifications in the theory of tensor products of field extensions, J. London Math. Soc., 15 (1977), 48-50.

[14] Z. Shmuely, The tensor product of distributive lattices, Algebra Universalis, 9 (1979), 281-296.

[15] P. Vamos, On the minimal prime ideals of a tensor product of two fields, Math. Proc. Camb. Phil. Soc. 84 (1978), 25-35. 